\title{
Existence of Periodic Properties of Solutions of Certain Au- tonomous Third Order Nonlinear Differential Equations
}

\section{Olutimo Akinwale L.}

Department of Mathematics, Lagos State University, Ojo. Lagos, Nigeria.

\section{Correspondence}

Olutimo Akinwale L., Department of Mathematics, Faculty of Science, Lagos State University, Nigeria.

Email:aolutimo@yahoo.com

\section{Funding information}

This research work is self sponsored.

\begin{abstract}
:
Introduction: It is well known that the periodic properties of solutions play a key role in characterizing the behavior of solutions of nonlinear differential equations. With reference to our observation in the relevant literature, work on the periodic properties of solutions for certain autonomous third-order nonlinear differential equations are very scarce.

Aims: In this work, we establish sufficient conditions that ensure the existence of periodic (or almost periodic) solutions of this class of differential equations.

Materials and Methods: The Lyapunov's second or direct method, a complete Lyapunov function was constructed and used to obtain our results.

Results: Sufficient Conditions were obtained for the existence of periodic and almost periodic solutions for certain autonomous third-order nonlinear differential equation.

Conclusion: The results extend and improve on some earlier results in the literature.

Keywords: Periodic solutions, Almost periodic solutions; Third order nonlinear differential equations, Lyapunov's method.
\end{abstract}

All co-authors agreed to have their names listed as authors.

This is an open access article under the terms of the Creative Commons Attribution License, which permits use, distribution and reproduction in any medium, provided the original work is properly cited.

(c) 2018 The Author. Journal of Research and Reviews in Science - JRRS, A Publication of Lagos State University 


\section{INTRODUCTION}

This paper considers the periodic or almost periodic solutions of the autonomous third-order differential equation

$$
\dddot{x}+\varphi(x, \dot{x}) \ddot{x}+g(\dot{x})+h(x)=p(t, x, \dot{x}, \ddot{x}),
$$

in which $\varphi, g, h$ and $p$ depend on the arguments displayed explicitly and dots denote differentiation with respect to t. Moreover, the existence and the uniqueness of solutions of (1) will be assumed.

Equations of the form (1) do arise in some aspect of applied sciences such as after effect, nonlinear oscillations, biological systems and equations with deviating arguments (see [1], [2] and [3]) and an effective method for studying the qualitative properties of solutions of such nonlinear equations is still the Lyapunov's direct method (see [4], [5], [6], [7], [8], [9], [10], [11], [12]). Many of these results on stability, boundedness, convergence of solutions exist for more general or special cases of (1) and are summarized in [13]. The search for periodic solutions and the examination of their behavior is of interest because of the mathematical description of nonlinear systems and the determination of periodic regime of real physical systems modeled into nonlinear differential equations. The periodic properties of solutions for some kind of nonlinear third-order scalar differential equations has been addressed by only a few, for example Andres [14], Ezeilo [15], Ezeilo [16], Ezeilo and Nkashama [17], Ogbu [18], Pliss [19] and Villari [20]. However, the methods employed by [14, 15, 16, $17,18,19]$ and $[20]$ were based on fixed point theorems, the Brouwer fixed-point theorem and the Lerray-Schauder fixed point theorem also referred to as the "non-Routh Hurwitz" direction in proving the existence of periodic solutions of thirdorder differential equations.

In this work, we consider a somewhat different approach to "non-Routh Hurwitz" direction in establishing the existence of periodic or almost periodic solutions of equation (1) if $p$ is periodic or almost periodic due to the presence of the perturbation $r$. To the best of our knowledge, results in the direction of Routh-Hurwitz do not exist! The problem, however, in using the Lyapunov method approach to establish the existence of periodic solutions is the difficulty in constructing a suitable complete Lyapunov function (see [4]). Our results will be in the direction of Routh Hurwitz and may be applied to the spatial discretion of some third-order differential equations (see [21]).

\section{MATERIAL AND METHODS}

\subsection{Definitions}

Definition 2.1 A continuous function $f: \mathbb{R} \rightarrow x$ is called almost periodic if for each $\varepsilon>0$ there exists $\ell(\varepsilon)>0$ such that every interval of length $\ell(\varepsilon)$ contains a number $\tau$ with property that

$$
|f(t+\tau)-f(t)|<\varepsilon \text { for each } t \in \mathbb{R} .
$$

Definition 2.2 A continuous function $f: \mathbb{R} \rightarrow x$ is said to be periodic with period $\omega$ for all $t \in \mathbb{R}$ such that

$$
f(t+\omega)=f(t) \text { for all } t \in \mathbb{R} .
$$

Assume now that $r$ is the perturbation such that $p$ is continuous function $p(t, x, \dot{x}, \ddot{x})$ is separable in the form

$$
p(t, x, \dot{x}, \ddot{x})=q(t)+r(t, x, \dot{x}, \ddot{x}),
$$

with $q(t)+r(t, 0,0,0)$ continuous in their respective arguments, where

$$
|q(t)|=\int_{0}^{t}|q(s)| \leq D_{1}, \quad D_{1}>0 .
$$

Our main result is the following

\subsection{Main result}

Theorem 2.3 Further to the basic assumptions imposed on the functions $\varphi, g, h$ and $p$ in equation (1). We also assume that $a, b, c, \delta_{o}$ are positive constants and $h(0)=0$, then the following conditions hold:

(i) $\varphi(x, y)>a, \frac{g(y)}{y} \geq b, h_{x}(x) \leq c$ and $a b-c>0$ for all
$x, y, z ;$

(ii) $\frac{h(x)}{x} \geq \delta_{o}$, for all $x \neq 0$;

(iii) $y \varphi_{x}(x, y) \leq 0$, for all $x, y$;

(iv) $p(t, x, y, z) \equiv q(t)+r(t, x, y, z)$ satisfies

$$
\begin{aligned}
& \left|r\left(t, x_{1}, y_{1}, z_{1}+q\right)-r\left(t, x_{2}, y_{2}, z_{2}+q\right)\right| \leq \\
& \phi\left\{\left|x_{1}-x_{2}\right|+\left|y_{1}-y_{2}\right|+\left|z_{1}-z_{2}\right|\right\} .
\end{aligned}
$$

for arbitrary $t$ and $x_{1}, x_{2}, y_{1}, y_{2}, z_{1}, z_{2} \in \mathbb{R}$, with $\phi(t)$ a continuous function satisfying

$$
\int_{-\infty}^{\infty} \phi^{\gamma}(t) d t<\infty
$$

for some constant $\gamma$ in the range $1 \leq \gamma \leq 2$. Suppose further that there exists a solution $x(t)$ of equation (1) such that

$$
|x(t)|^{2}+|\dot{x}(t)|^{2}+|\ddot{x}(t)|^{2} \leq D_{2} \text {. }
$$

Then,

1. If $q(t)$ is almost periodic and $r(t, x, \dot{x}, \ddot{x})$ is almost periodic in $t$, for $|x(t)|^{2}+|\dot{x}(t)|^{2}+|\ddot{x}(t)|^{2} \leq D_{2}$, then $x(t)$ is almost periodic in $t$. 
2. If $q(t)$ and $r(t, x, \dot{x}, \ddot{x})$ are periodic in $t$, with period $\omega$, for $|x(t)|^{2}+|\dot{x}(t)|^{2}+|\ddot{x}(t)|^{2} \leq D_{2}$, then $x(t)$ is periodic with period $\omega$. tem

Now, let equation (1) be replaced with the equivalent sys-

$$
\begin{aligned}
\dot{x} & =y \\
\dot{y} & =z+q \\
\dot{z} & =-\varphi(x, y) z-g(y)-h(x)+p(t, x, y, z+q) \\
& -\varphi(x, y) q .
\end{aligned}
$$

\subsection{Preliminary results}

Let $(x, y, z)$ be any solution of system (2). Our main tool is the following scalar function, defined by

$$
V=V_{1}+V_{2},
$$

where $V_{1}$ and $V_{2}$ are given by

$$
\begin{aligned}
2 V_{1} & =2 \int_{0}^{x} h(\xi) d \xi+2 \int_{0}^{y} \varphi(x, \sigma) d \sigma+2 \alpha \int_{0}^{y} g(\sigma) d \sigma \\
& +\alpha z^{2}+2 y z+2 \alpha y h(x)
\end{aligned}
$$

and

$$
\begin{aligned}
2 V_{2} & =\beta \ell b x^{2}+2 a \int_{0}^{x} h(\xi) d \xi+2 a \int_{0}^{y} \varphi(x, \sigma) d \sigma \\
& +2 \alpha \int_{0}^{y} g(\sigma) d \sigma+z^{2}+2 \beta \ell x y+2 a y z \\
& +2 y h(x)+2 \beta \ell x z,
\end{aligned}
$$

$\alpha>0$ is a fixed constant chosen such that

$$
\frac{1}{a}<\alpha<\frac{b}{c}
$$

and $0<\beta<1$ chosen such that

$$
\begin{gathered}
\beta<\min \left\{\frac{a b-c}{\ell\left[a+\delta_{o}^{-1}\left(\frac{g(y)}{y}-b\right)^{2}\right]}, \frac{1}{a},\right. \\
\left.\frac{a \alpha-1}{a b \alpha}, \frac{\delta_{o}(a \alpha-1)}{\ell[\varphi(x, y)-a]^{2}}\right\} .
\end{gathered}
$$

The function $V$ can be re-arranged as follows:

$$
\begin{aligned}
2 V_{1} & =\left\{2 \int_{0}^{x} h(\xi) d \xi-\frac{\alpha}{b} h^{2}(x)\right\}+\alpha b\left\{y+\frac{h(x)}{b}\right\}^{2} \\
& +\left\{2 \int_{0}^{y} \varphi(x, \sigma) d \sigma-\alpha^{-1} y^{2}\right\}+\alpha\left\{z+\alpha^{-1} y\right\}^{2} \\
& +\alpha\left\{\int_{0}^{y} g(\sigma) d \sigma-b y^{2}\right\}
\end{aligned}
$$

and

$$
\begin{aligned}
2 V_{2} & =\beta \ell(b-\beta \ell) x^{2}+a\left\{2 \int_{0}^{x} h(\xi) d \xi-\ell^{-1} h^{2}(x)\right\} \\
& +\ell\left\{a^{\frac{1}{2}} y+\ell^{-1} a^{-\frac{1}{2}} h(x)\right\}^{2}+\left\{2 \int_{0}^{y} g(\sigma) d \sigma-\ell a^{-1} y^{2}\right\} \\
& +a\left\{\int_{0}^{y} \varphi(x, \sigma) d \sigma-a y^{2}\right\}+\{\beta \ell x+a y+z\}^{2} .
\end{aligned}
$$

The term

$$
2 \int_{0}^{x} h(\xi) d \xi-\frac{\alpha}{b} h^{2}(x),
$$

in the re-arrangement of $2 V_{1}$ becomes

$$
2 \int_{0}^{x}\left(1-\frac{\alpha}{b} h_{\xi}(\xi)\right) h(\xi) d \xi-\frac{\alpha}{b} h^{2}(0),
$$

using the hypotheses (i) and (ii) of Theorem 1, we get

$$
2 \int_{0}^{x} h(\xi) d \xi-\frac{\alpha}{b} h^{2}(x) \geq\left(1-\frac{\alpha}{b} c\right) \delta_{o} x^{2} .
$$

Similarly, the term

$$
2 \int_{0}^{x} h(\xi) d \xi-\ell^{-1} h^{2}(x),
$$

in the re-arrangement of $2 V_{2}$ becomes

$$
2 \int_{0}^{x} h(\xi) d \xi-\ell^{-1} h^{2}(x) \geq\left(1-\frac{c}{\ell}\right) \delta_{o} x^{2} .
$$

Combining these estimates, we obtain for

$$
\begin{aligned}
V & \geq\left\{\left(1-\frac{\alpha}{b} c\right) \delta_{o}+\beta \ell(b-\beta \ell)+\left(1-\frac{c}{\ell}\right) \delta_{o}\right\} x^{2} \\
& +\left\{\left(a-\frac{1}{\alpha}-\beta \ell\right)+\left(b-\frac{\ell}{a}\right)\right\} y^{2} \\
& +\alpha\left(z+\frac{1}{\alpha} y\right)^{2}+(\beta \ell x+a y+z)^{2},
\end{aligned}
$$

where $\alpha, \beta$ satisfy (4) and (5) if we chose $\ell=a b$.

Thus, there exists a constant $\delta_{1}$ small enough such that

$$
V \geq \delta_{1}\left(x^{2}+y^{2}+z^{2}\right),
$$

where

$$
\begin{aligned}
\delta_{1} & =\min \left\{\left(1-\frac{\alpha}{b} c\right) \delta_{o}+\beta \ell(b-\beta \ell)+\left(1-\frac{c}{\ell}\right) \delta_{o} ;\left(a-\frac{1}{\alpha}-\beta \ell\right)\right. \\
& \left.+\left(b-\frac{\ell}{a}\right) ;(\alpha+1)\right\} .
\end{aligned}
$$

Also, from (3) and by Schwartz's inequality, we have

$$
V \leq \delta_{2}\left(x^{2}+y^{2}+z^{2}\right),
$$


where

$$
\begin{array}{r}
\delta_{2}=\max \left\{1+a+\delta_{o}(1+\alpha)+\beta(2+b) ; 2+a(a+2)+\alpha(b+1)+\beta \ell+b ;\right. \\
\alpha+2+a+\beta \ell\} . \quad \text { whe }
\end{array}
$$

Hence, $V$ is positive definite and satisfies

$$
\delta_{1}\left(x^{2}+y^{2}+z^{2}\right) \leq V \leq \delta_{2}\left(x^{2}+y^{2}+z^{2}\right) .
$$

Furthermore, along any solution $(x(t), y(t), z(t))$ of (2), we have

$$
\frac{d}{d t} V(x, y, z)=\dot{V}
$$

given by

$$
\begin{aligned}
\dot{V} & =(1+a) y \int_{0}^{y} \varphi_{x}(x, \sigma) d \sigma+(1+\alpha) h_{x}(x) y^{2} \\
& -(1+a) \frac{g(y)}{y} y^{2} \\
& -[\varphi(x, y)-a] z^{2}-\alpha \varphi(x, y) z^{2}-\beta \ell\left\{\frac{g(y)}{y}-b\right\} x y+z^{2} \\
& -\beta \ell \frac{h(x)}{x} x^{2}-\beta \ell[\varphi(x, y)-a] x z+a \beta \ell y^{2} \\
& +(1+\alpha) \frac{h(x)}{x} x q(t)+\left\{(1+\alpha) \frac{g(y)}{y} y-\beta \ell y\right\} q(t) \\
& +(1-\alpha a) z q(t) \\
& +[\beta \ell x+(1+a) y+(1+\alpha) z] r(t, x, y, z+q) .
\end{aligned}
$$

By the hypotheses of the Theorem, we have

$$
\begin{aligned}
\dot{V} & \leq(1+\alpha) c y^{2}-(1+a) b y^{2}-\alpha a z^{2}+z^{2} \\
& -\beta \ell\left\{\frac{g(y)}{y}-b\right\} x y \\
& -\beta \ell[\varphi(x, y)-a] x z+\beta \ell \delta_{o} x^{2}+a \beta \ell y^{2}-a z^{2} \\
& +\left[(1+\alpha) \delta_{o} x+(b(1+\alpha)-\beta \ell) y+(1-\alpha a) z\right] q(t) \\
& +[\beta \ell x+(1+a) y+(1+\alpha) z] r(t, x, y, z+q) .
\end{aligned}
$$

It is obvious that

$$
\begin{aligned}
\dot{V}= & -\frac{1}{2} \beta \ell \delta_{o} x^{2}-\left[a b-c-\beta \ell\left(a+\delta_{o}^{-1}\left(\frac{g(y)}{y}-b\right)^{2}\right] y^{2}\right. \\
& -(b-\alpha c) y^{2}-\left[a \alpha-1-\beta \ell \delta_{o}^{-1}(\varphi(x, y)-a)^{2}\right] z^{2}-a z^{2} \\
& -\frac{1}{4} \beta \ell \delta_{o}\left[x+2 \delta_{o}^{-1}\left(\frac{g(y)}{y}-b\right) y\right]^{2} \\
& -\frac{1}{4} \beta \ell \delta_{o}\left[x+2 v^{-1}(\varphi(x, y)-a) z\right]^{2} \\
& +\left[(1+\alpha) \delta_{o} x+(b(1+\alpha)-\beta \ell) y+(1-\alpha a) z\right] q(t) \\
& +[\beta \ell x+(1+a) y+(1+\alpha) z] r(t, x, y, z+q)
\end{aligned}
$$

If $\beta$ satisfies (5), we have that

$$
\begin{aligned}
\dot{V} \leq & -\frac{1}{2} \beta \ell \delta_{o} x^{2}-(b-\alpha c) y^{2}-a z^{2} \\
& +\left[(1+\alpha) \delta_{o} x+(b(1+\alpha)-\beta \ell) y+(1-\alpha a) z\right] q(t) \\
& +[\beta \ell x+(1+a) y+(1+\alpha) z] r(t, x, y, z+q) .
\end{aligned}
$$

It follows that

$$
\begin{aligned}
\dot{V} \leq & -\delta_{3}\left(x^{2}+y^{2}+z^{2}\right)+\delta_{4}\left(x^{2}+y^{2}+z^{2}\right)^{\frac{1}{2}} \\
& +\delta_{5}\left(x^{2}+y^{2}+z^{2}\right)^{\frac{1}{2}}|r(t, x, y, z+q)|
\end{aligned}
$$

where

$$
\delta_{3}=\min \frac{1}{2}\left\{\beta \ell \delta_{o}, 2(b-\alpha c), 2 a\right\}
$$

$$
\delta_{4}=\max \sqrt{3} D_{1}\left\{\delta_{o}(1+\alpha), b(1+\alpha)-\beta \ell, 1-\alpha a\right\},
$$

and

$$
\delta_{4}=\max \sqrt{3}\{\beta \ell, 1+a, 1+\alpha\}
$$

Thus,

$$
\begin{aligned}
\dot{V} \leq & -\delta_{3}\left(x^{2}+y^{2}+z^{2}\right) \\
& +\delta_{6}\left(x^{2}+y^{2}+z^{2}\right)^{\frac{1}{2}}[r(t, x, y, z+q)+1]
\end{aligned}
$$

where

$$
\delta_{6}=\max \left\{\delta_{4}, \delta_{5}\right\}
$$

So that since

$$
\begin{gathered}
|r(t, x, y, z+q)| \leq \delta_{6} \phi(t)\left[\left(x^{2}+y^{2}+z^{2}\right)^{\frac{1}{2}}+1\right] \\
\dot{V} \leq \quad-\quad \delta_{3}\left(x^{2}+y^{2}+z^{2}\right)+\delta_{6} \phi(t)\left(x^{2}+y^{2}+z^{2}\right) \\
+\delta_{6} \phi(t)\left(x^{2}+y^{2}+z^{2}\right)^{\frac{1}{2}}
\end{gathered}
$$

By (iv) of Theorem 1 and (6), we have

$$
\dot{V} \leq-\left(\delta_{7}-\delta_{8} \phi(t)\right) V+\delta_{9} V^{\frac{1}{2}}
$$

where $\delta_{7}=\frac{\delta_{3}}{\delta_{2}}, \quad \delta_{8}=\frac{\delta_{5}}{\delta_{1}} \quad \delta_{9}=\frac{\delta_{4}}{\delta_{1}}$. that

Following the argument used in [5] it can be further verified

$$
\dot{V} \leq-\delta_{10}\left(x^{2}+y^{2}+z^{2}\right)+\delta_{11} \phi(t)\left(x^{2}+y^{2}+z^{2}\right)^{\frac{1}{2}}|\theta|,(7)
$$
where $\theta=r\left(t, x_{1}, y_{1}, z_{1}+q\right)-r\left(t, x_{2}, y_{2}, z_{2}+q\right)$ and $\delta_{10}, \delta_{11}$ are finite constants.

\section{RESULTS}

\subsection{Proof of Theorem 1}

Consider the function

$U(t)=V(x(t-\tau)-x(t), y(t-\tau)-y(t), z(t-\tau)-z(t))$ 
where $\mathrm{V}$ is the function defined in (3) with $x, y, z$ replaced by $(x(t+\tau)-x(t)),(y(t+\tau)-y(t))$ and $(z(t+\tau)-z(t))$ respectively. Then, by (6) we have positive constants $D_{3}$ and $D_{4}$ such that

$$
D_{3} S(t) \leq U(t) \leq D_{4} S(t)
$$

where

$S(t)=\left\{|x(t+\tau)-x(t)|^{2}+|y(t+\tau)-y(t)|^{2}+|z(t+\tau)-z(t)|^{2}\right\}$

Differentiating $U(t)$ along the system (2), we get as in (7),

$$
\begin{aligned}
\dot{U}(t) & \leq-\delta_{10}\left\{|x(t+\tau)-x(t)|^{2}+|y(t+\tau)-y(t)|^{2}\right. \\
& \left.+|z(t+\tau)-z(t)|^{2}\right\} \\
& +\delta_{11}\left\{|x(t+\tau)-x(t)|^{2}+|y(t+\tau)-y(t)|^{2}\right. \\
& \left.+|z(t+\tau)-z(t)|^{2}\right\}^{\frac{1}{2}}|\theta|
\end{aligned}
$$

where $\theta=r((t+\tau), x(t), y(t), z(t)+q(t+\tau)-r(t, x, y, z+q)$ with $\delta_{10}$ and $\delta_{11}$ being finite constants.

Inequality (10) can be arranged as

$$
\begin{aligned}
\dot{U}(t) & \leq-\delta_{10}\left\{|x(t+\tau)-x(t)|^{2}+|y(t+\tau)-y(t)|^{2}\right. \\
& \left.+|z(t+\tau)-z(t)|^{2}\right\} \\
& +\delta_{12}\left\{|x(t+\tau)-x(t)|^{2}+|y(t+\tau)-y(t)|^{2}\right. \\
& \left.+|z(t+\tau)-z(t)|^{2}\right\}^{\frac{1}{2}}|\theta| \\
& +\delta_{13}\left\{|x(t+\tau)-x(t)|^{2}+|y(t+\tau)-y(t)|^{2}\right. \\
& \left.+|z(t+\tau)-z(t)|^{2}\right\}^{\frac{1}{2}} \\
& \times \mid r(t+\tau), x(t), y(t), z(t)+q(t+\tau)-r(t, x(t), y(t), z
\end{aligned}
$$

Since the perturbation $r$ is uniformly almost periodic in $\mathrm{t}$. Then, given arbitrary $\varepsilon>0$, we can find $\tau>0$ such that $\mid q(t+\tau)-$ $q(t) \mid \leq \ell \varepsilon^{2}$

$|r(t+\tau), x(t), y(t), z(t)+q(t+\tau)-r(t, x(t), y(t), z+q)| \leq \ell \varepsilon^{2}$

where $\ell$ is a constant whose value will be determined later. Thus, (11) becomes

$$
\dot{U}(t) \leq-\delta_{10} S(t)+\delta_{12} S^{\frac{1}{2}}|\theta|+\delta_{13} S^{\frac{1}{2}}(t) \ell \varepsilon^{2}
$$

By (iv) of Theorem 1,

$\left\{|x(t+\tau)-x(t)|^{2}+|y(t+\tau)-y(t)|^{2}+|z(t+\tau)-z(t)|^{2}\right\}^{\frac{1}{2}} \leq \mathcal{D}_{2}$

(13) becomes,

$$
\dot{U}(t)+\delta_{10} S(t) \leq \delta_{12}^{\frac{1}{2}}|\theta|+\delta_{13} \mathcal{D}_{2} \ell \varepsilon^{2} .
$$

Let $\gamma$ be any constant such that $1 \leq \gamma \leq 2$ and set $m=1-\frac{1}{2} \gamma$, so that $0 \leq m \leq \frac{1}{2}$.

Then, (14) becomes

$$
\dot{U}+\delta_{10} S(t) \leq \delta_{12} S^{m} U^{*}+\delta_{13} \mathcal{D}_{2} \ell \varepsilon^{2}
$$

and $U^{*}=S^{\left(\frac{1}{2}-m\right)}\left(|\theta|-\delta_{10} \delta_{12}^{-1} S^{\frac{1}{2}}(t)\right)$.

\section{We consider two cases}

(i) $|\theta| \leq \delta_{10} \delta_{12}^{-1} S^{\frac{1}{2}}$ and

(ii) $|\theta|>\delta_{10} \delta_{12}^{-1} S^{\frac{1}{2}}$

separately, we find that in either case, there exists some constants $\delta_{14}>0$ such that $U^{*} \leq \delta_{14}|\theta|^{2(1-m)}$. Thus, (15) becomes

$$
\frac{d U}{d t}+\delta_{10} S \leq \delta_{15} S^{m} \phi^{2(1-m)} S^{(1-m)} U(t)+\delta_{13} \mathcal{D}_{2} \ell \varepsilon^{2}
$$

where $\delta_{15} \geq 2 \delta_{12} \delta_{14}$. Using (9) on $U$, we obtain

$$
\frac{d U}{d t}+\left(\left(\delta_{16}-\delta_{17}\right) \phi^{\gamma}(t)\right) U(t) \leq \delta_{13} \mathcal{D}_{2} \ell \varepsilon^{2}
$$

where $\delta_{16}, \delta_{17}$ as positive constants.

On integrating (16) from $t_{o}$ to $t\left(t \geq t_{o}\right)$, we obtain

$$
\begin{aligned}
W(t) & \left.\leq \delta_{18} U\left(t_{1}\right) \exp \left\{-\delta_{16}\left(t-t_{o}\right)\right\}+\delta_{17} \int_{t_{o}}^{t} \phi^{\gamma}(s) d(s)\right\} \\
& +\delta_{19} \ell \varepsilon^{2}
\end{aligned}
$$

where $\delta_{18}=\frac{\delta_{12}}{\delta_{10}}$ and $\delta_{19}=\frac{\delta_{18} \delta_{13} \mathcal{D}_{2}}{\delta_{16}}$.

If

$$
\int_{t_{o}}^{t} \phi^{\gamma}(s) d(s)<\delta_{16} \delta_{17}^{-1}\left(t-t_{o}\right)
$$

then, the exponential index remains negative for all $\left(t-t_{o}\right) \geq$ 0 . As $t=\left(t-t_{o}\right) \rightarrow \infty$ and that $U\left(t_{o}\right)$ is finite in (17), we have that

$$
U(t) \leq \delta_{19} \ell \varepsilon^{2} \text { for any } t
$$

Since $U(t)$ satisfies (9), we get

$$
U(t) \leq D_{3}^{-1} \delta_{19} \ell \varepsilon^{2} .
$$

Also, by (9), we have that

$$
\begin{aligned}
& |x(t+\tau)-x(t)|+|y(t+\tau)-y(t)|+|z(t+\tau)-z(t)| \leq \\
& \left(\frac{3 \ell \delta_{19}}{D_{3}}\right)^{\frac{1}{2}} \varepsilon .
\end{aligned}
$$

Choosing $\ell=\frac{D_{3}}{3 \delta_{19}}$ in (18), we have

$|x(t+\tau)-x(t)|+|y(t+\tau)-y(t)|+|z(t+\tau)-z(t)| \leq \varepsilon,(19)$

where $\tau$ is chosen to satisfy (12) is relatively dense and hence (19) implies that the solutions $(x(t), y(t), z(t))$ or equivalently $x(t), \dot{x}(t), \ddot{x}(t)$ of (1) are uniformly almost periodic in $\mathrm{t}$. 

that

To show that the solutions are also periodic, we assume

$$
\begin{aligned}
q(t+\omega) & =q(t) \\
r(t+\omega, x(t), y(t), z(t)+q(t)) & =r(t, x(t), y(t), z(t)),
\end{aligned}
$$

for $\left(x^{2}+y^{2}+z^{2}\right) \leq D_{2}$.

Since the perturbation $r(t, x, y, z+q)$ has period $\omega$ in $\mathrm{t}$, we replace $\tau$ in the definition of $U(t)$ with $\omega$. The terms in the left hand side of (12) is identically zero, thus we may have inequality (19) as

$|x(t+\omega)-x(t)|+|y(t+\omega)-y(t)|+|z(t+\omega)-z(t)| \leq 0$.

Thus,

$|x(t+\omega)-x(t)|+|y(t+\omega)-y(t)|+|z(t+\omega)-z(t)|=0$.

which implies that

$$
x(t+\omega)=x(t) \text { and } y(t+\omega)=y(t) \quad z(t+\omega)=z(t)
$$

That is, $x(t), y(t), z(t)$ are periodic in $\mathrm{t}$ with period $\omega$.

\section{CONCLUSION}

Analysis of nonlinear systems literary shows that Lyapunov's theory in Periodic properties of solutions is rarely scarce. The second Lyapunov's method allows to predict the periodic behavior of solutions of sufficiently complicated nonlinear physical system. The solutions of third-order autonomous differential equation (1) are periodic and almost periodic uniformly in $x, \dot{x}$ and $\ddot{x}$ according to Lyapunov's theory if (4) and (5) hold as $t \rightarrow \infty$.

\section{ACKNOWLEDGEMENTS}

The author would like to thank the reviewer for his helpful comments and suggestions.

\section{CONFLICTS OF INTERESTS}

The author declare no conflict of interest.

\section{AUTHORS' CONTRIBUTIONS}

The study was designed and conducted by Olutimo Akinwale. $\mathrm{He}$ also wrote the manuscript.

\section{CONSENT}

Consent form has been approved by the author.

\section{REFERENCES}

[1] Afuwape AU, Omari P, Zanalin F. Nonlinear pertubations of differential operators with nontrivial kernel and applications to third-order periodic boundary problems. J. Math. Anal. Appl. 1989;143:35-36.

[2] Cronin J. Some mathematics of biological oscillations. SIAM Rev. 1997;19:100-137.

[3] Rauch LL. Oscillations of a third-order nonlinear autonomous system: in: contributions to the Theory of nonlinear oscillations. Ann of Math. Stud. 1950;20:39-88.

[4] Chukwu EN. On the boundedness of solutions of third order differential equations. Ann. Mat. Pura Appl. 1975;155(4):123-149.

[5] Ezeilo JOC. An elementary proof of a boundedness theorem for a certain third order differential equation. J. Lond. Math Soc. 1963:11-16.

[6] Ezeilo JOC. New properties of the equation $\dddot{x}+a \ddot{x}+b \dot{x}+$ $h(x)=p(t, x, \dot{x}, \ddot{x})$ for certain special values of the incrementary ratio $y^{-1} h(x+y)-h(x)$, quations differentielles et functionelles non linaires ( Actes Conference Internat.) "Equa-Dff. 73", Brussels/Louvain-la-Neuve, Herman, Paris; 1973:447-462.

[7] Olutimo AL. Ultimate boundedness of solutions for certain third-order nonlinear differential equations, Mathematical Theory and Modeling. 2016;6(3):136-142.

[8] Olutimo AL, Akinwole FO. Stability and boundedness of solutions of certain non-autonomous third-order nonlinear differential equations. Journal of Applied Mathematics and Physic. 2016;4:149-155.

[9] Omeike MO. New result in the ultimate boundedness of solutions of a third-order nonlinear ordinary differential equation. J. of Inequalities in Pure and Applied mathematics. 2008;9:Article 15.8.

[10] Omeike MO. Further results for the solutions of certain third-order differential equations. Nonlinear Analysis. 2007;67:3394-3400.

[11] Omeike MO, Olutimo AL, Oyetunde OO. The boundedness of solutions of certain nonlinear third order ordinary differential equations. J. Nig. Math. Soc. 2012;31:49-54.

[12] Tunc C. The boundedness of solutions of nonlinear third order differential equations. Nonlinear Dynamics and Systems Theory. 2010;10(1):97-102. 
[13] Reissig R, Sansone G, Conti R. Nonlinear differential equations of higher order. Translated from the German. Leyden: Noordhoff International Publishing; 1974.

[14] Andres J. Periodic boundary value problem for nonlinear equations of the third order. Math Slovaca. 1985;25(3):305-309.

[15] Ezeilo JOC. Further result on the existence of periodic solutions of the equation $\dddot{x}+\psi(\dot{x}) \ddot{x}+\phi(x) \dot{x}+$ $v(x, \dot{x}, \ddot{x})=p(t, x, \dot{x}, \ddot{x})$ with a bound $v$. Atti. Accad.Naz.Lincei Rend.Cl.Sci.Fis.Mat.Natur. 1978;55:5157.

[16] Ezeilo JOC. Periodic solutions of third order differential equations in the past twenty-five years or so, invited paper presented at 2nd Pan African Congress of Afri. math. Union, March, University of Jos, Nigeria; 1986.

[17] Ezeilo JOC, Nkashama N. Non uniform, non resonance and existence of periodic solutions of some third order nonlinear ordinary differential equations. Annals T.M.A. 1986;12:1029-1046.

[18] Ogbu HM. Existence of periodic solutions for a certain nonlinear third order differential equation. Pacific Journal of Science and Technology. 2006;7(2):120-125.

[19] Pliss VA. Study of nonlinear differential equations of the third order. Dovl. Akad. Nauk SSSR. 1956;111:11781180 .

[20] Villari G. Criteria existeza di soluzioni periodic per particulari class di equezioni differential del Ter'ordine nonlineari. Mathematica Cataria. 1964;19:96-112.

[21] Hugo L. Existence of bounded solutions of a secondorder system with dissipation. Journal of Math. Analysis and Aplication. 1999;237(1):288-302. 